\title{
CARIOLOGIA DE ALGUMAS ESPÉCIES DE PTERIDÓFITAS OCORRENTES NO NORDESTE DO BRASIL ${ }^{1}$
}

\author{
Adriana Buarque Marcon² \\ Iva Carneiro Leão Barros ${ }^{2}$ \\ Marcelo Guerra ${ }^{2}$
}

Recebido em 23/01/2001. Aceito em 16/09/2001

\begin{abstract}
RESUMO - (Cariologia de algumas espécies de pteridófitas ocorrentes no nordeste do Brasil). Foram analisadas citogeneticamente, através de técnicas de coloração convencional, sete espécies de pteridófitas homosporadas e três heterosporadas, pertencentes a oito famílias, sendo essas as primeiras contagens cromossômicas para essas espécies no Brasil. Dois parâmetros principais foram analisados no presente estudo: número cromossômico e tipos de núcleos interfásicos. Em algumas espécies, a morfologia cromossômica e a presença de satélites também foram avaliadas. De todas as espécies analisadas, duas não se tem conhecimento de contagens anteriores: Selaginella convoluta (Arn.) Spring $(2 \mathrm{n}=24)$ e Thelypteris serrata (Cav.) Alston $(2 \mathrm{n}=72)$, as quais apresentaram números já esperados para os gêneros. Nas demais espécies os números cromossômicos observados foram: Acrostichum aureum L., 2n=60; A. danaeifolium Langsd. \& Fisch., 2n=60; Blechnum occidentale L., 2n=124; Cyathea microdonta (Desv.) Domin, 2n=138; Hemionitis palmata $\mathrm{L}$., 2n=60; Marsilea quadrifolia $\mathrm{L} ., 2 \mathrm{n}=40$; Osmunda cinnamomea L., $\mathrm{n}=22$ e Selaginella kraussiana (Kunze) A. Braun., 2n=20. A única alteração cariológica observada foi a presença de um tetravalente no único indivíduo analisado de $O$. cinnamomea. A estrutura do núcleo interfásico foi mais densamente reticulada, e mais homogeneamente corada nas espécies homosporadas, enquanto nas heterosporadas essa estrutura foi mais variável, apresentando quase sempre cromômeros ou cromocentros em número variado.
\end{abstract}

Palavras-chave - pteridófitas, número cromossômico, núcleos interfásicos

\begin{abstract}
Karyology of some pteridophytes species occurring in the northeast of Brazil). Seven species of homosporous and three of heterosporous pteridophytes belonging to eight families, were cytogenetically analysed using conventional staining techniques. This is the first count for Brazilian populations of these species. Two karyological parameters were analysed: chromosome number and structure of interphase nucleus. Chromosome morphology and occurrence of satellites have also been evaluated in some species. Prior reports on chromosome counts are unkown for two of the species analysed: Selaginella convoluta (Arn.) Spring $(2 n=24)$ and Thelypteris serrata (Cav.) Alston $(2 \mathrm{n}=72)$, which presented numbers already expected for their genera. The other species analysed had the following chromosome numbers: Acrostichum aureum L., $2 \mathrm{n}=60$; A. danaeifolium Langsd. \& Fisch., 2n=60; Blechnum occidentale L., 2n=124; Cyathea microdonta (Desv.) Domin, 2n=138; Hemionitis palmata L., $2 \mathrm{n}=60$; Marsilea quadrifolia L., 2n=40; Osmunda cinnamomea L., n=22 and Selaginella kraussiana (Kunze)
\end{abstract}

\footnotetext{
Auxílio CNPq e FACEPE.

2 Departamento de Botânica, CCB, Universidade Federal de Pernambuco, Rua Nelson Chaves s/n, Cidade Universitária, CEP 50670-420, Recife, PE, Brasil (mguerra@ufpe.br; abmarcon@uol.com.br)
} 
A. Braun., $2 \mathrm{n}=20$. The only cytogenetical irregularity observed was the presence of a tetravalent in a single individual analysed of $O$. cinnamomea. The structure of the interphase nuclei was more densely reticulated and homogeneously stained in the homosporous species, while in the heterosporous ones, it was more variable, almost always presenting different numbers of chromomers or chromocenteres.

Key words - pteridophytes, chromosome number, interphase nuclei

\section{Introdução}

O estudo citotaxonômico em pteridófitas teve início com Irene Manton em 1950 (Walker 1979). Posteriormente, diversas floras pteridofíticas começaram a ser estudadas citotaxonomicamente, como a do Sri Lanka (Manton \& Sledge 1954), Jamaica (Walker 1966) e Trinidad (Walker 1985). O avanço da investigação citológica da flora pteridofítica contribuiu para o entendimento dos mecanismos de evolução do grupo. As contagens cromossômicas evidenciaram a poliploidia como o principal mecanismo citológico envolvido na evolução do grupo, sendo refletida nos altos números cromossômicos encontrados nas pteridófitas. Além disso, mecanismos de hibridização interespecíficas mostraram-se de ocorrência comum, contribuindo, juntamente com a poliploidização, para o surgimento de novas espécies.

Apesar de serem bem estudadas citotaxonomicamente em vários outros países, no Brasil, o estudo citológico em pteridófitas restringe-se a um único trabalho elaborado por Tryon et al. (1975) em 11 espécies pertencentes a quatro gêneros ocorrentes no estado do Amazonas. Visando contribuir para o conhecimento citológico das pteridófitas brasileiras, foi realizada a contagem cromossômica de 10 espécies ocorrentes no Nordeste. Os dados obtidos foram comparados em relação aos existentes na literatura.

\section{Material e métodos}

As espécies analisadas foram coletadas nos Estados de Pernambuco, Paraíba e Bahia. A Tabela 1 apresenta a relação das espécies analisadas, com respectivas famílias, seguindo a classificação de Tryon \& Tryon (1982), bem como os locais de coleta, os números cromossômicos observados e as contagens cromossômicas prévias. Parte do material foi herborizado e inserido no acervo do herbário Geraldo Mariz, da Universidade Federal de Pernambuco (UFP). Outra parte do material foi cultivada no Jardim Experimental do Laboratório de Citogenética Vegetal do Departamento de Botânica da Universidade Federal de Pernambuco (UFPE) para a análise citológica. Para a análise mitótica, foram coletadas pontas de raízes em crescimento ativo, pré-tratadas em 8 -hidroxiquinoleína $0,002 \mathrm{M}$ por $1 \mathrm{~h}$ à temperatura ambiente, seguida de $23 \mathrm{~h}$ a $8^{\circ} \mathrm{C}$. Posteriormente, as raízes foram fixadas em Carnoy (etanol-ácido acético 3:1) por 2-24h à temperatura ambiente, e conservadas em freezer. Para a análise meiótica, foram coletados esporângios jovens, fixados diretamente em Carnoy, por $2 \mathrm{~h}$ à temperatura ambiente, e conservados em freezer.

Para a análise mitótica, as raízes foram lavadas em água destilada, 2 vezes por 5 min cada lavagem, hidrolisadas em $\mathrm{HCl} 5 \mathrm{~N}$ por 30 min e esmagadas em ácido acético $45 \%$. Nas espécies em que as raízes eram muito duras, foi primeiramente feita uma digestão da parede celular em celulase $2 \%$-pectinase $20 \%$ por $6 \mathrm{~h}$ a $37^{\circ} \mathrm{C}$, antes da hidrólise. Em seguida o material foi corado com hematoxilina $1 \%$ e montado em Entellan (Guerra 1999). Na preparação das lâminas para a análise meiótica, os esporângios foram esmagados diretamente em carmim acético a $2 \%$. As medições dos cromossomos foram feitas com o auxílio de compasso de ponta seca e régua. A nomenclatura dos cromossomos foi baseada em Levan et al. (1964). A 
Tabela 1. Lista das espécies analisadas, com os respectivos locais de coleta, número cromossômico, contagens prévias e referências.

\begin{tabular}{|c|c|c|c|c|c|}
\hline \multirow[t]{2}{*}{ Família/Espécie } & \multirow[t]{2}{*}{ Local de coleta } & \multirow[t]{2}{*}{$\mathrm{n}$} & \multirow[t]{2}{*}{$2 n$} & Contagens prévias & \multirow[t]{2}{*}{ Referências* } \\
\hline & & & & $2 n$ & \\
\hline
\end{tabular}

\begin{tabular}{|c|c|c|c|c|c|c|}
\hline $\begin{array}{l}\text { Blechnaceae } \\
\text { Blechnum occidentale L. }\end{array}$ & Maraial (PE) & - & 124 & $62,64,40^{\mathrm{II}}+44^{\mathrm{I}}$, ca. 93 & 92,124 & $a-j$ \\
\hline $\begin{array}{l}\text { Cyatheceae } \\
\text { Cyathea microdonta (Desv.) } \\
\text { Domin }\end{array}$ & Paulista (PE) & - & 138 & 69 & - & $\mathrm{h}$ \\
\hline $\begin{array}{l}\text { Marsileaceae } \\
\text { Marsilea quadrifolia L. }\end{array}$ & Recife (PE) & - & 40 & 20 & 40 & $f, i$ \\
\hline $\begin{array}{l}\text { Osmundaceae } \\
\text { Osmunda cinnamomea } \mathrm{L} . \\
\text { Pteridaceae }\end{array}$ & Rio de Contas (BA) & $20^{\mathrm{II}}+1^{\mathrm{IV}}$ & - & 22 & - & d, e, i \\
\hline Acrostichum aureum L. & $\begin{array}{l}\text { Ipojuca (PE) } \\
\text { Rio Tinto (PB) }\end{array}$ & $\begin{array}{l}30 \\
30\end{array}$ & $\begin{array}{l}60 \\
60\end{array}$ & & 120 & $\mathrm{i}, \mathrm{l}, \mathrm{m}, \mathrm{n}, \mathrm{o}$ \\
\hline $\begin{array}{l}\text { Acrostichum danaeifolium } \\
\text { Langsd. \& Fisch. }\end{array}$ & $\begin{array}{l}\text { Paulista (PE) } \\
\text { Utinga (BA) }\end{array}$ & 30 & $\begin{array}{l}60 \\
60\end{array}$ & 30 & 60 & $\mathrm{~h}$ \\
\hline Hemionitis palmata $\mathrm{L}$. & Maraial (PE) & - & 60 & 30 & - & $\mathrm{b}, \mathrm{e}, \mathrm{i}, \mathrm{p}, \mathrm{q}$ \\
\hline $\begin{array}{l}\text { Sela ginellaceae } \\
\text { Selaginella convoluta (Arn.) } \\
\text { Spring. }\end{array}$ & Itapororoca (PB) & - & 22 & - & - & - \\
\hline $\begin{array}{l}\text { Selaginella kraussiana } \\
\text { (Kunze) A. Braun. }\end{array}$ & Recife (PE) & - & 20 & - & - & $\mathrm{r}$ \\
\hline $\begin{array}{l}\text { Thelypteridaceae } \\
\text { Thelypteris serrata (Cav.) }\end{array}$ & Paulista (PE) & 36 & 72 & - & - & - \\
\hline
\end{tabular}

* a- Goldblatt 1981; b- Smith \& Mickel 1977; c- Goldblatt \& Johnson 1990; d- Fabri 1965; e- Walker 1966; f- Goldblatt 1988; g- Goldblatt \& Johnson 1991; h- Walker 1985; i- Fabri 1963; j- Jarret et al. 1968; 1- Kawakami 1979; m- Kawakami 1980; n- Kawakami 1982; o- Roux 1993; p- Manton \& Sledge 1954; q- Moore 1974; r- Moore 1973.

identificação dos tipos de núcleos interfásicos foi baseada na classificação de Guerra (1985). As melhores células foram analisadas em microscópio Leica DMRB, utilizando a lente objetiva de 100x e fotografadas com filme Kodak Imagelink HQ ASA 25, copiados em papel Kodak Kodabromide F3.

\section{Resultados e discussão}

As três espécies heterosporadas analisadas (Marsilea quadrifolia, Selaginella convoluta e Selaginella kraussiana), apresentaram núcleo do tipo semi-reticulado, diferenciando-se quanto à densidade da cromatina $\mathrm{e}$ ao tamanho dos cromocentros (Fig. 1). Em Selaginella, notou-se a presença de numerosos cromômeros ou pequenos cromocentros em S. kraussiana, enquanto em $S$. convoluta essas estruturas foram mais difíceis de serem visualizadas devido a maior condensação da cromatina. Por outro lado, todas as espécies homosporadas apresentaram núcleo interfásico do tipo reticulado, com retículo de cromatina denso e fortemente corado (Fig. 2 e 3). Em angiospermas, os tipos de núcleos interfásicos são freqüentemente relacionados ao tamanho cromossômico e à quantidade de DNA nuclear (Guerra 1985). Takamiya (1993) chamou a atenção para a importância da análise dos tipos de núcleos interfásicos na citotaxonomia das pteridófitas. Aparentemente, há maior varia- 
bilidade de organização da cromatina entre as espécies heterosporadas. Análise detalhada desse parâmetro poderá contribuir significantemente para a compreensão da evolução cariológica do grupo.

Marsilea quadrifolia apresentou 40 cromossomos bem pequenos, cujo tamanho variou de 0,8 a $2,5 \mu \mathrm{m}$ (Fig. 4). Trabalhos anteriores nesta espécie (Fabri 1963; Goldblatt 1988), também reportaram $2 \mathrm{n}=40$ e números básicos $x=20$ e 22 para o gênero, tratando-se, portanto, de espécie diplóide.

Selaginella convoluta apresentou 24 cromossomos, com tamanho cromossômico variando entre 0,8 e $2,0 \mu \mathrm{m}$ (Fig. 5). Em S. kraussiana, a variação do tamanho cromossômico entre os 20 cromossomos que compõem o cariótipo foi de 0,7 a $1,1 \mu \mathrm{m}$ (Fig. 6). Os números cromossômicos encontrados em $S$. convoluta e em $S$. kraussiana coincidiram com dados de outras espécies do mesmo gênero (Goldblatt 1988; Takamiya 1993; Goldblatt \& Johnson 1996). Os números básicos mais prováveis do gênero são $x=8,9,10$ e 11 (Fabri 1963; 1965), portanto, essas espécies devem ser diplóides.

Entre as pteridófitas homosporadas, sete espécies foram analisadas: Acrostichum aureum, A. danaeifolium, Blechnum occidentale, Hemionitis palmata, Osmunda cinnamomea, Thelypteris serrata e Cyathea microdonta. As duas espécies do gênero Acrostichum, A. aureum e A. danaeifolium, apresentaram $2 \mathrm{n}=60$, em concordância com contagens anteriores (Tab. 1). Entretanto, nenhuma variação numérica foi observada no presente trabalho, como reportado para populações de A. aureum (Kawakami 1982; Roux 1993; Nakato 1996). O tamanho cromossômico nessa última espécie variou de 4,9 a 8,3 $\mu \mathrm{m}$. O conjunto cromossômico mostrouse composto principalmente por cromossomos subtelocêntricos, dois pares dos quais eram satelitados (Fig. 7). Acrostichum danaeifolium apresentou menor variação de tamanho cromossômico em relação a A. aureum $(4,1$ a
$6,6 \mu \mathrm{m})$, mas mostrou morfologia cromossômica e número de satélites similares (Fig. 8). A descrição do cariótipo dessas espécies foi detalhada por Marcon et al. (dados inéditos). $\mathrm{Na}$ análise meiótica, os indivíduos analisados de ambas as espécies, mostraram meiose regular, com 30 bivalentes.

Cyathea microdonta com $2 \mathrm{n}=138$, apresentou o maior número cromossômico do presente trabalho. O tamanho cromossômico do conjunto variou entre $1,6 \mu \mathrm{m}$ e o maior $3,3 \mu \mathrm{m} \mathrm{e}$ nenhum satélite foi observado (Fig. 9). Nessa espécie não foi encontrado nenhum registro de análise mitótica, entretanto, Walker (1985) registrou o número meiótico $\mathrm{n}=69$. O número cromossômico observado coincidiu com o de outras espécies do gênero (Tryon \& Tryon 1982).

Em Hemionitis palmata, o número cromossômico encontrado foi $2 \mathrm{n}=60$, com predominância de cromossomos subtelocêntricos, alguns pares submetacêntricos e o menor par metacêntrico. O tamanho dos cromossomos variou de 1,6 a $2,5 \mu \mathrm{m}$ (Fig. 10). Não foi encontrada nenhuma análise mitótica prévia dessa espécie, entretanto, diversos autores (Manton \& Sledge 1954; Fabri 1963; Walker 1966; Smith \& Mickel 1977; Moore 1974; Walker 1985; Goldblatt \& Johnson 1990; 1991) descreveram seu número meiótico, $n=30$, e o número básico do gênero, $\mathrm{x}=30$.

A análise mitótica de Blechnum occidentale mostrou a presença de 124 cromossomos, em concordância com contagens anteriores (Fabri 1963; Walker 1985). O gênero Blechnum apresenta série de números básicos de $\mathrm{x}=28$ a $\mathrm{x}=36$ (Walker 1985), sendo essa espécie, com $2 \mathrm{n}=124$, provavelmente um tetraplóide com número básico $\mathrm{x}=31$. A morfologia cromossômica não foi muito clara, parecendo apresentar cromossomos subtelocêntricos e telocêntricos em sua maioria e menor quantidade de submetacêntricos e metacêntricos (Fig. 11). Foi observado ao menos um par telocêntrico 


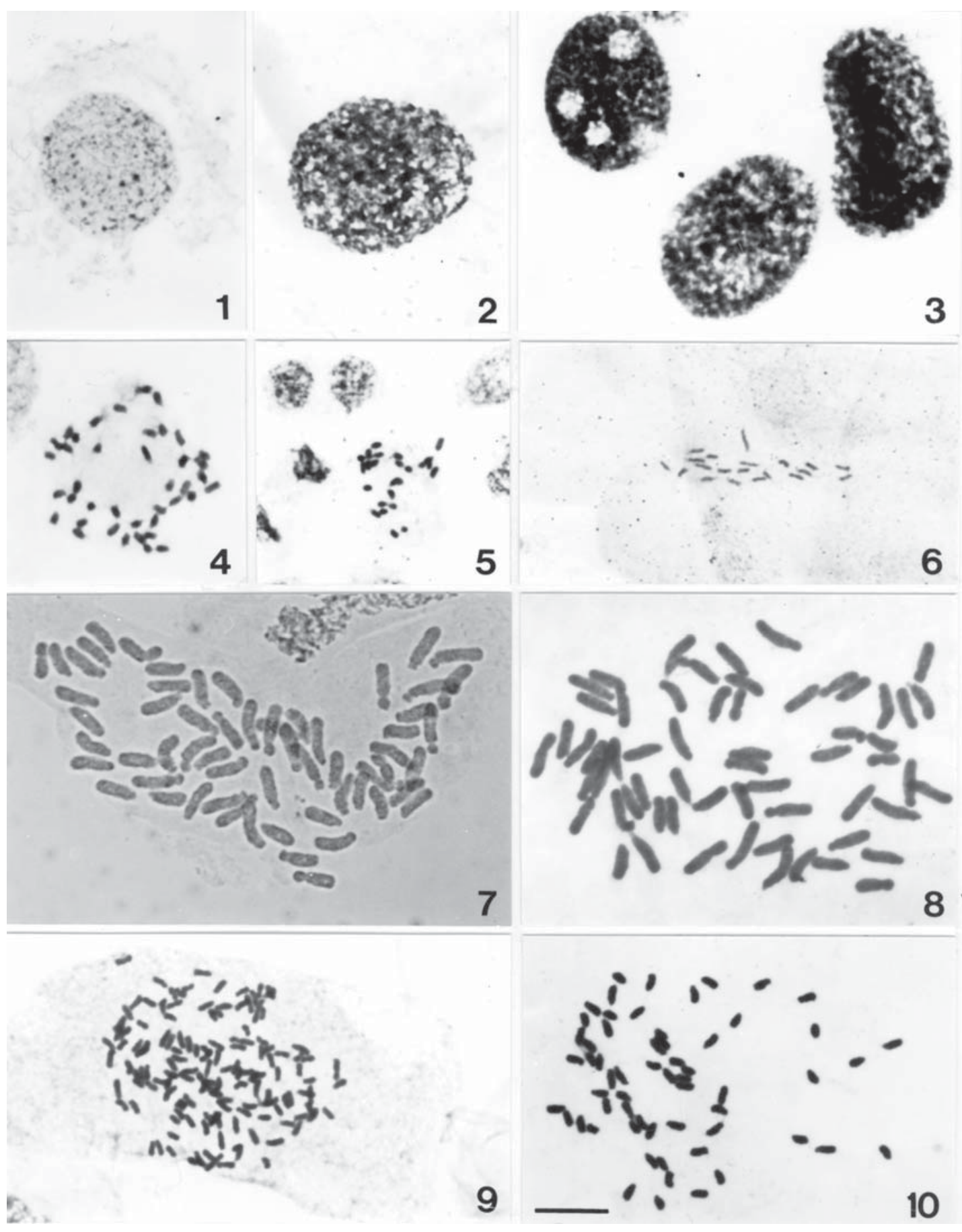

Figuras 1-10. Núcleos interfásicos e metáfases de pteridófitas heterosporadas $(1,4,5,6)$ e homosporadas $(2,3,7,8,9$, 10). 1. Núcleo semi-reticulado com pequenos cromocentros de Marsilea quadrifolia. 2-3. Núcleo reticulado em Thelypteris serrata (2) e Blechnum occidentale (3). Observe em um dos núcleos de B. occidentale, a presença de quatro núcléolos. 4-10. Cromossomos metafásicos de M. quadrifolia (4) com $2 \mathrm{n}=40$, Selaginella convoluta (5) com $2 \mathrm{n}=24, S$. kraussiana (6) com $2 \mathrm{n}=20$, Acrostichum aureum (7) $\operatorname{com} 2 \mathrm{n}=60$, A. danaeifolium (8) com $2 \mathrm{n}=60$, Cyathea microdonta (9) com $2 \mathrm{n}=138$ e Hemionitis palmata $(10)$ com $2 \mathrm{n}=60$. Barra corresponde a $10 \mu \mathrm{m}$. 


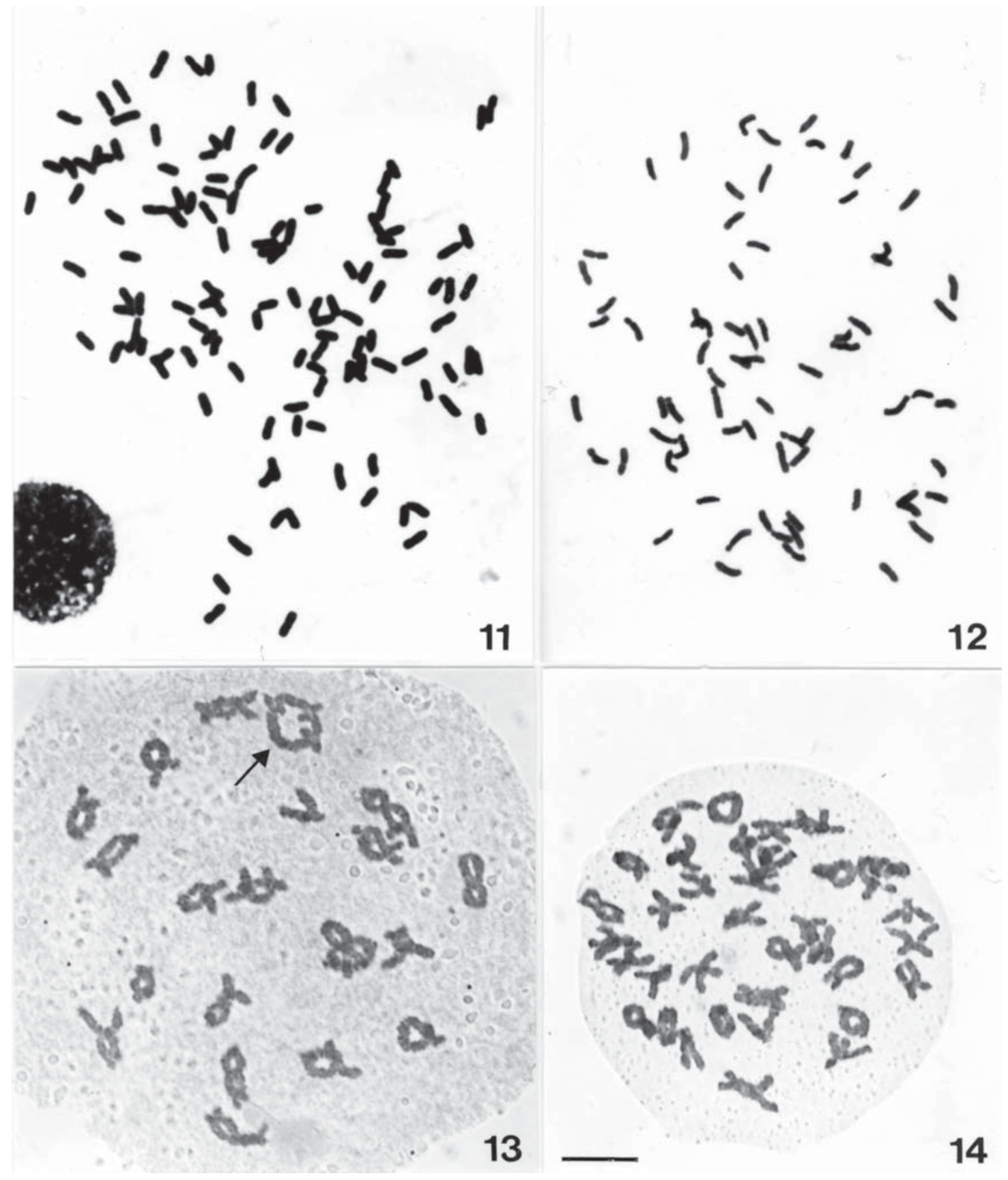

Figuras 11-14. Células mitóticas e meióticas de pteridófitas homosporadas. 11-12. Metáfase mitótica de Blechnum occidentale (11) com $2 \mathrm{n}=124$ e de Thelypteris serrata (12) com $2 \mathrm{n}=72$. 13-14. Diacinese de Osmunda cinnamomea (13) $\operatorname{com~} \mathrm{n}=20^{\mathrm{II}}+1^{\mathrm{IV}}$ e de $T$. serrata (14) com $\mathrm{n}=36$. A seta em $O$. cinnamomea indica o tetravalente. Barra corresponde a $10 \mu \mathrm{m}$. 
satelitado. Walker (1985) também evidenciou apenas um par telocêntrico satelitado em um indivíduo de $B$. occidentale coletado na Jamaica e fez referência à grande quantidade de cromossomos metacêntricos e submetacêntricos do cariótipo. O menor cromossomo do conjunto apresentou $2,5 \mu \mathrm{m}$ e o maior, 4,1 $1 \mu \mathrm{m}$.

Thelypteris serrata apresentou $2 \mathrm{n}=72$, com cromossomos geralmente subtelocêntricos a telocêntricos, distinguindo-se um par satelitado metacêntrico e outro telocêntrico. O tamanho dos cromossomos variou de 2,9 a 4,5 $\mu \mathrm{m}$ (Fig. 12). A análise meiótica evidenciou 36 bivalentes e um ciclo meiótico normal (Fig. 14). Não foi encontrado nenhum registro citogenético da espécie na literatura, embora esse mesmo número cromossômico já tenha sido encontrado em outras espécies de Thelypteris (Tryon \& Tryon 1982). Considerando que os números básicos do gênero provavelmente são $\mathrm{x}=27,30$, 31, 32, 34, 35, 36 (Fabri 1963), essa espécie deve ser diplóide.

A análise meiótica de Osmunda cinnamomea mostrou cromossomos grandes, com $n=22$, sendo 20 bivalentes e um tetravalente (Fig. 13). A presença de um tetravalente sugere que tenha ocorrido uma translocação entre dois cromossomos não-homólogos, gerando um polimorfismo para a translocação na população. O número encontrado no presente estudo coincide com contagens anteriores para a espécie e para o gênero, que apresenta número cromossômico estável, sem disploidia ou poliploidia (Walker 1966), e número básico $\mathrm{x}=22$ (Fabri 1963; 1965).

Os dados do presente trabalho mostram uma estabilidade dos números cromossômicos nas espécies de pteridófitas ocorrentes no Brasil e em outras regiões. Ao lado disso, esses resultados confirmam a tendência comum nas pteridófitas de apresentarem números mais altos nas homosporadas e mais baixos nas heterosporadas. Os altos números encontrados nas espécies homosporadas são indicativos de paleopoliploidia, um tipo de poliplóide que deve ter surgido num passado remoto, caracterizado pela ausência de espécies próximas diplóides (Guerra 2000). A maior freqüência de poliploidia nas espécies com homosporia é atribuída à alta incidência de fecundação intragametofítica (autofecundação) encontrada nesses organismos (Klekowski 1979). Isso parece se refletir também nos números básicos mais elevados das homosporadas, estabelecendo diferença cariológica marcante em relação às heterosporadas, que apresentam números básicos mais baixos (Cubas 1990).

\section{Referências bibliográficas}

Cubas, P. 1990. Procesos citogenéticos de especiación en Pteridophyta. Anales Jardín Botánico de Madrid 46: 519-531.

Fabri, F. 1963. Primo supplemento alle tavole cromosomiche delle Pteridophyta di Alberto Chiarugi. Caryologia 16: 237-335.

Fabri, F. 1965. Secondo supplemento alle tavolle cromosomiche delle Pteridophyta di Alberto Chiarugi. Caryologia 18: 675-731.

Goldblatt, P. (Ed.). 1981. Index to plant chromosome numbers 1975-1978. Missouri Botanical Garden, Saint Louis.

Goldblatt, P. (Ed.). 1988. Index to plant chromosome numbers 1984-1985. Missouri Botanical Garden, Saint Louis.

Goldblatt, P. \& Johnson, D. E. (Ed.). 1990. Index to plant chromosome numbers 1986-1987. Missouri Botanical Garden, Saint Louis.

Goldblatt, P. \& Johnson, D. E. (Ed.). 1991. Index to plant chromosome numbers 1988-1989. Missouri Botanical Garden, Saint Louis.

Goldblatt, P. \& Johnson, D. E. (Ed.). 1996. Index to plant chromosome numbers 1992-1993. Missouri Botanical Garden, Saint Louis.

Guerra, M. 1985. Estrutura e diversificação de núcleos interfásicos em plantas. Pp. 137-153. In: M. L. R. Aguiar-Perecin; P. S. Martins \& G. Bandel (Eds.). Tópicos de citogenética e evolução de plantas. Sociedade Brasileira de Genética, Ribeirão Preto.

Guerra, M. 1999. Hematoxylin: a simple, multiple-use dye for chromosome analysis. Genetic and molecular biology 22: 77-80.

Guerra, M. 2000. Chromosome number variation and evolution in Monocots. Pp. 127-136. In: K. L. Wilson \& D. A. Morrison (Eds.). Monocots: systematics and evolution. CSIRO, Melbourne. 
Jarret, F. M.; Manton, I. \& Roy, S. K. 1968. Cytological and taxonomic notes on a small collection of living ferns from Galapagos. Kew Bulletin 22: 475-480.

Kawakami, S. 1979. Karyomorphological studies on Japanese Pteridaceae I - Dennstaedtia, Microlepia, Sphenomeris, Pteris. Bulletin of Aichi University of Education (Natural Science) 28: 85-104.

Kawakami, S. 1980. Karyomorphological studies on Japanese Pteridaceae II - Pteris, Acrostichum, Cheilanthes, Onychium. Bulletin of Aichi University of Education (Natural Science) 29: 129-150.

Kawakami, S. 1982. Karyomorphological studies on Japanese Pteridaceae IV - Discussion. Bulletin of Aichi University of Education (Natural Science) 31: 175-186.

Klekowski, E. J. Jr. 1979. The genetics and reproductive biology of ferns. Pp. 133-169. In: A. F. Dyer (Ed.). The Experimental Biology of Ferns. Academic Press, London.

Levan, A.; Fredga, K. \& Sandberg, A. 1964. Nomenclature for centromeric position on chromosomes. Hereditas 52: 201-220.

Manton, I. \& Sledge, W. A. 1954. Observations of the cytology and taxonomy of the Pteridophyte flora of Ceylon. Philosophical Transactions of the Royal Society of London 238: 127-185.

Moore, R. J. (Ed.). 1973. Index to plant chromosome numbers 1967-1971. Regnum Vegetabile 90, Utrecht.
Moore, R. J. (Ed.). 1974. Index to plant chromosome numbers 1972. Regnum Vegetabile 91, Utrecht.

Nakato, N. 1996. Notes on chromosomes of Japanese Pteridophytes (4). The Journal of Japanese Botany 71: 163-167.

Roux, J. P. 1993. A new cytotype for Acrostichum aureum. Bothalia 23: 75.

Smith, A. R. \& Mickel, J. T. 1977. Chromosome counts for Mexican ferns. Brittonia 29: 391-398.

Takamiya, M. 1993. Comparative karyomorphology and interrelationships of Selaginella in Japan. Journal of Plant Research 106: 149-166.

Tryon, A. F.; Bautista, H. P. \& Araújo, I. da S. 1975. Chromosome studies of Brazilian ferns. Acta Amazonica 5: 35-43.

Tryon, R. M. \& Tryon, A. 1982. Ferns and allied plants - with special reference to tropical America. Springer-Verlag, New York.

Walker, T. G. 1966. A cytotaxonomic survey of the Pteridophytes of Jamaica. Transactions of the Royal Society Edinburgh 66: 169-237.

Walker, T. G. 1979. The cytogenetics of ferns. Pp. 87-132. In: A. F. Dyer (Ed.). The experimental biology of ferns. Academic Press, London.

Walker, T. G. 1985. Cytotaxonomic studies of the ferns of Trinidad 2. The citology and taxonomic implications. Bulletin of the British Museum (Natural History) 13: 149-249. 